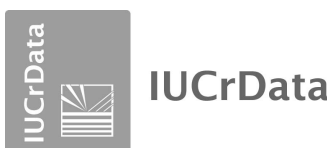

ISSN 2414-3146

Received 12 May 2018

Accepted 12 June 2018

Edited by R. J. Butcher, Howard University, USA

Keywords: crystal structure; cobalt; nitrate; 2,2'bipyridine; supramolecular interactions.

CCDC reference: 1848987

Structural data: full structural data are available from iucrdata.iucr.org

\section{fac-Triaqua $\left(2,2^{\prime}\right.$-bipyridine- $\left.\kappa^{2} N, N^{\prime}\right)($ nitrato- $\kappa O)$ - cobalt(II) chloride}

\author{
Mouhamadou Birame Diop, ${ }^{a *}$ Libasse Diop $^{\mathrm{a}}$ and Sylvain Bernès ${ }^{\mathrm{b}}$
}

aLaboratoire de Chimie Minérale et Analytique, Département de Chimie, Faculté des Sciences et Techniques, Université Cheikh Anta Diop, Dakar, Senegal, and ${ }^{\mathbf{b}}$ Instituto de Física, Benemérita Universidad Autónoma de Puebla, Av. San Claudio y 18 Sur, 72570 Puebla, Pue., Mexico. *Correspondence e-mail: mouhamadoubdiop@gmail.com

The asymmetric unit of the title complex, $\left[\mathrm{Co}\left(\mathrm{NO}_{3}\right)\left(\mathrm{C}_{10} \mathrm{H}_{8} \mathrm{~N}_{2}\right)\left(\mathrm{H}_{2} \mathrm{O}\right)_{3}\right] \mathrm{Cl}$, consists of a chloride anion and a complex cation, which is built on a monodentate nitrate anion, three water molecules and one bidentate 2,2'bipyridine molecule, coordinated to a $\mathrm{Co}^{\mathrm{II}}$ cation, in a distorted octahedral geometry. The water molecules are arranged in a facial geometry, and serve as donors for hydrogen bonding. Acceptor sites in the crystal are chloride ions and one $\mathrm{O}$ atom of the coordinating nitrate ion. A three-dimensional framework is formed, based on $\mathrm{O}-\mathrm{H} \cdots \mathrm{O}$ and $\mathrm{O}-\mathrm{H} \cdots \mathrm{Cl}$ contacts.
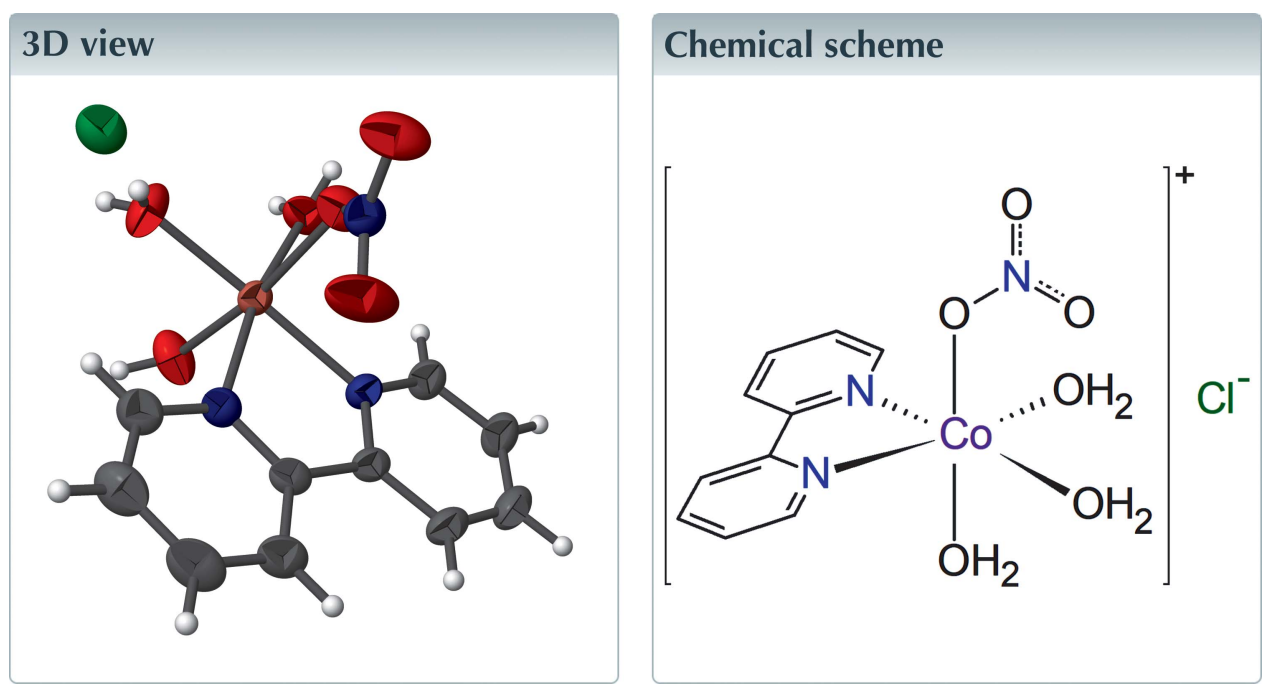

\section{Structure description}

As a component of several enzymes, vitamins, proteins and nucleic acids, pyridine derivatives have a main role in many biological systems. Some complexes containing 2,2'-bipyridine (bipy) have been reported to present antibacterial activity (El-Hamid et al., 2017; Lu et al., 2015; Segura et al., 2014), including cobalt complexes (Buriez et al., 2006; Gu et al., 2017). It can be expected that the combination of the high bactericidal activity of bipy and a cobalt cation may lead to a compound of interest. In this dynamic, we have initiated the study of the interaction between $\left[\mathrm{Na}_{3} \mathrm{Co}\left(\mathrm{NO}_{2}\right)_{6}\right]$, dimethylammonium chloride and bipy. The complex reported herein results from a redox process over the cobalt complex used as starting material, when the reaction is carried out in acetone and in a non-controlled atmosphere: the nitrite anion $\mathrm{NO}_{2}{ }^{-}$behaves as a reducing agent to reduce $\mathrm{Co}^{\mathrm{III}}$ to $\mathrm{Co}^{\mathrm{II}}$, and is in turn oxidized to form nitrate ions $\mathrm{NO}_{3}{ }^{-}$. A stable cation complex $\left[\mathrm{Co}(\text { bipy })\left(\mathrm{OH}_{2}\right)_{3}\left(\mathrm{NO}_{3}\right)\right]^{+}$is then formed, which crystallizes as a chloride salt in presence of $\left[\mathrm{NH}_{2} \mathrm{Me}_{2}\right]^{+} \mathrm{Cl}^{-}$.

The asymmetric unit of the title compound (Fig. 1) consists of a chloride anion and a complex cation containing a chelating 2,2'-bipyridine molecule, a monocoordinating 


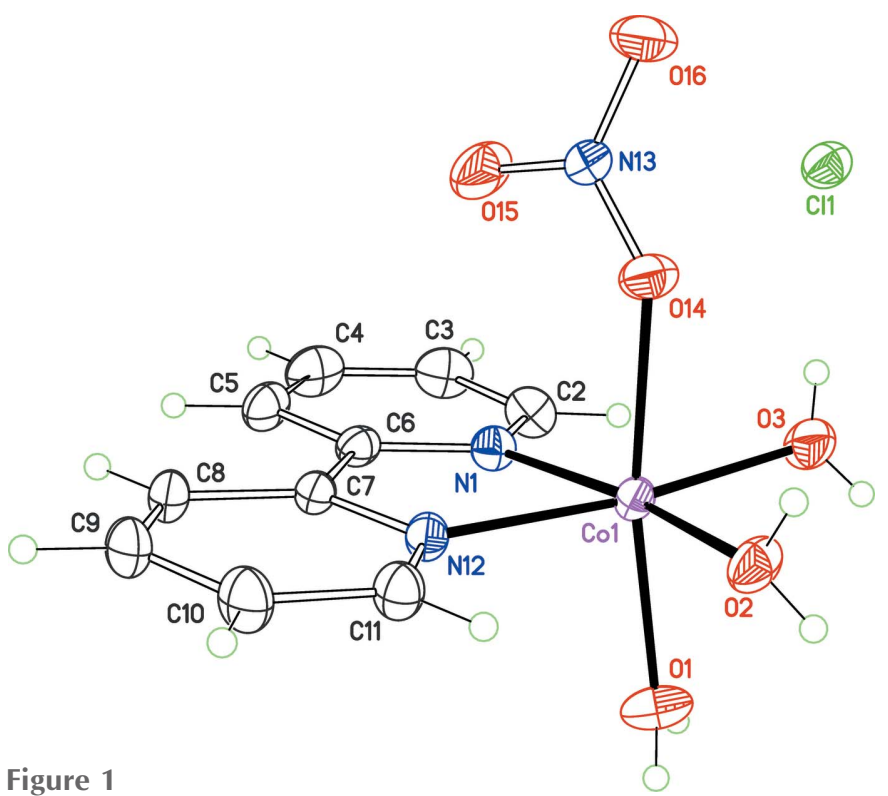

The structure of the complex cation and the anion in the title compound, with displacement ellipsoids for non- $\mathrm{H}$ atoms at the $30 \%$ probability level.

nitrate anion and three water molecules to complete the octahedral coordination sphere around the cobalt cation. Coordination bond lengths are in the small range 2.0300 (16)2.1769 (15) $\AA$, indicating that the ligand field should be small enough to stabilize a high spin $3 d^{7}$ metal configuration, reflected in the limited Jahn-Teller tetragonal distortion. Using the octahedral symmetry measure defined by Alvarez et al. (2002), $S\left(O_{h}\right)=5.39 \Delta^{2}-0.33|\Delta|$, where $\Delta$ is the difference between long and short distances, we compute $S\left(O_{h}\right)=0.07$ for the cation, close to the measure expected for an ideal octahedral field, $S\left(O_{h}\right)=0$. The coordinating water molecules have a facial geometry, with the longest $\mathrm{Co}-\mathrm{OH}_{2}$ bond displayed by the axial water molecule O1 (Abboud et al., 1996; Johnson et al., 2015). The nitrate ion is placed trans to this water molecule, forming an angle $\mathrm{O} 1-\mathrm{Co} 1-\mathrm{O} 14$ of $170.47(7)^{\circ}$. The equatorial plane includes the chelating bipy ligand and two water molecules, at normal distances (Xiao, 2006; Gong et al., 2012). The planarity of this $\left[\mathrm{CoO}_{2} \mathrm{~N}_{2}\right]$ core is confirmed by the sum of the four cis angles, $360.0(3)^{\circ}$. The distortion from the octahedral symmetry results essentially from the bipy bite angle, $\mathrm{N} 1-\mathrm{Co} 1-\mathrm{N} 12=77.37(6)^{\circ}$. The metal is displaced by $0.04 \AA$ from the equatorial mean plane. A similar arrangement was observed with other cations $\left[M(\text { bipy })\left(\mathrm{OH}_{2}\right)_{3}\left(\mathrm{NO}_{3}\right)\right]^{+}$ using different transition metals, $M=\mathrm{Mn}$ (Zhang et al., 2002), $M=\mathrm{Ni}$ (Walmsley et al., 1989; Rujiwatra et al., 2012), $M=\mathrm{Cu}$ (Mathews \& Manohar, 1991) and $M=\mathrm{Zn}$ (Harrowfield et al., 2017).

Coordinating water molecules are oriented in such a way that all $\mathrm{O}-\mathrm{H}$ groups serve as donors to form weak hydrogen bonds with chloride ions and atom $\mathrm{O} 16$ of the nitrate ion (Table 1). Each complex cation is then directly linked to four neighbours through intermolecular $\mathrm{O}-\mathrm{H} \cdots \mathrm{O}$ bonds, and the supramolecular structure is extended to a three-dimensional framework through $R_{4}^{2}(8)$ ring motifs based on $\mathrm{O}-\mathrm{H} \cdots \mathrm{Cl}$
Table 1

Hydrogen-bond geometry $\left(\AA,^{\circ}\right)$.

\begin{tabular}{|c|c|c|c|c|}
\hline$D-\mathrm{H} \cdots A$ & $D-\mathrm{H}$ & $\mathrm{H} \cdots A$ & $D \cdots A$ & $D-\mathrm{H} \cdots A$ \\
\hline $\mathrm{O} 1-\mathrm{H} 11 \cdots \mathrm{Cl} 1^{\mathrm{i}}$ & $0.85(4)$ & $2.33(4)$ & $3.1560(19)$ & $166(4)$ \\
\hline $\mathrm{O} 1-\mathrm{H} 12 \cdots \mathrm{O} 16^{\mathrm{ii}}$ & $0.73(3)$ & $2.13(3)$ & $2.825(2)$ & $160(4)$ \\
\hline $\mathrm{O} 2-\mathrm{H} 21 \cdots \mathrm{Cl} 1^{\mathrm{iii}}$ & $0.81(4)$ & $2.39(4)$ & 3.1833 (18) & $167(3)$ \\
\hline $\mathrm{O} 2-\mathrm{H} 22 \cdots \mathrm{Cl} 1^{\mathrm{ii}}$ & $0.74(4)$ & $2.37(4)$ & $3.1088(16)$ & $177(4)$ \\
\hline $\mathrm{O} 3-\mathrm{H} 31 \cdots \mathrm{Cl} 1$ & $0.74(4)$ & $2.36(4)$ & 3.100 (2) & $176(3)$ \\
\hline $\mathrm{O} 3-\mathrm{H} 32 \cdots \mathrm{O} 16^{\mathrm{i}}$ & $0.77(4)$ & $1.98(4)$ & $2.745(2)$ & $178(4)$ \\
\hline
\end{tabular}

Symmetry codes: (i) $-x, y+\frac{1}{2},-z+\frac{1}{2}$; (ii) $x,-y+\frac{1}{2}, z+\frac{1}{2}$; (iii) $-x, y-\frac{1}{2},-z+\frac{1}{2}$.

bonds (Fig. 2). The relative orientation of the cations in the crystal resulting from this supramolecular structure does not prevent $\pi-\pi$ interactions between bipy ligands: two cations related by inversion have their bipy parts placed parallel, with a separation between the mean planes of $3.375 \AA$.

\section{Synthesis and crystallization}

All chemicals were purchased from Aldrich Company and used as received. Sodium hexanitrocobaltate(III) [ $\left.\mathrm{Na}_{3} \mathrm{Co}\left(\mathrm{NO}_{2}\right)_{6}\right] \quad(125 \mathrm{mg}, 0.309 \mathrm{mmol})$, dimethylammonium chloride $(25 \mathrm{mg}, 0.309 \mathrm{mmol})$ and 2,2'-bipyridine $(48 \mathrm{mg}$, $0.309 \mathrm{mmol}$ ) were mixed together at room temperature in slightly hydrated acetone. The resulting solution was stirred for about two h at $303 \mathrm{~K}$. After a week of slow evaporation at room temperature, orange crystals suitable for X-ray crystallographic analysis were obtained.

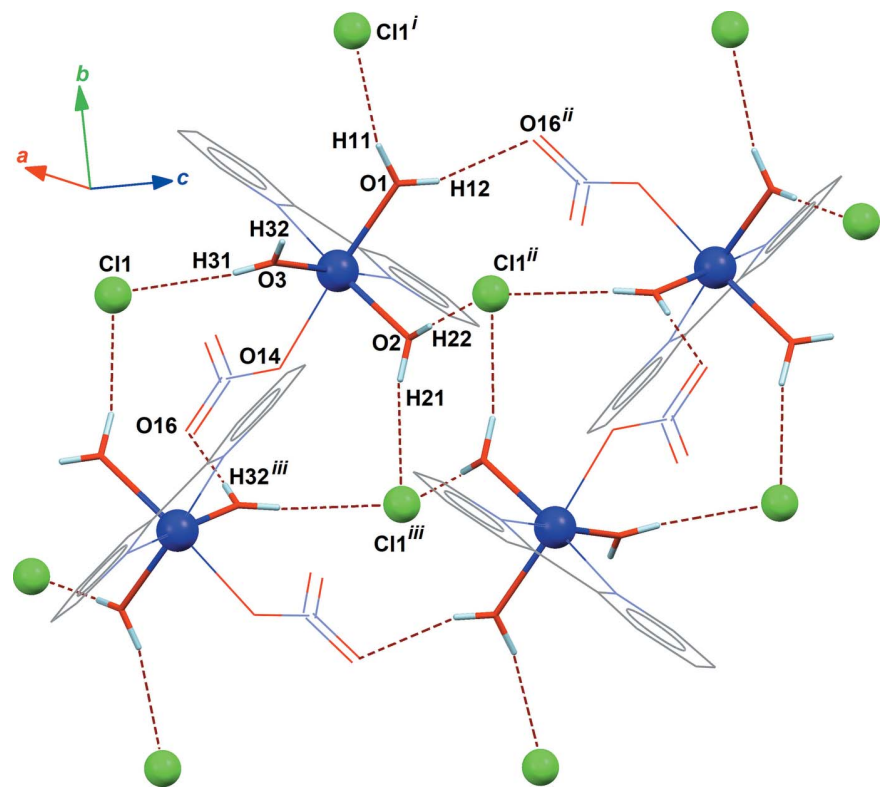

Figure 2

Part of the crystal structure of the title compound showing four cations and ten chloride forming hydrogen bonds (dashed lines). $\mathrm{H}$ atoms belonging to the bipy ligands have been omitted for clarity. The asymmetric unit contains the upper-left cation and Cl1. Symmetry codes: (i) $-x, \frac{1}{2}+y, \frac{1}{2}-z$; (ii) $x, \frac{1}{2}-y, \frac{1}{2}+z$; (iii) $-x,-\frac{1}{2}+y, \frac{1}{2}-z$. 


\section{Refinement}

Crystal data, data collection and structure refinement details are summarized in Table 2. Intensities were collected at high resolution $\left[(\sin \theta) / \lambda=0.92 \AA^{-1} ; d=0.54 \AA\right]$.

\section{Funding information}

The authors acknowledge the Cheikh Anta Diop University of Dakar (Senegal) and CONACyT, Mexico for financial support (Project 268178).

\section{References}

Abboud, K. A., Palenik, R. C. \& Palenik, G. J. (1996). Acta Cryst. C52, 2994-2996.

Alvarez, S., Avnir, D., Llunell, M. \& Pinsky, M. (2002). New J. Chem. 26, 996-1009.

Buriez, O., Moretto, L. M. \& Ugo, P. (2006). Electrochim. Acta, 52, 958-964.

El-Hamid, S. M. A., El-Demerdash, R. S., Arafat, H. F. H. \& Sadeek, S. A. (2017). J. Mol. Struct. 1149, 613-625.

Gong, Y., Li, J., Qin, J. \& Lin, J. (2012). CrystEngComm, 14, 58625869.

Gu, A., Xiang, W., Wang, T., Gu, S. \& Zhao, X. (2017). Solar Energy, 147, 126-132.

Harrowfield, J. M., Kim, Y. \& Skelton, B. W. (2017). CSD communication, CCDC 990117.

Johnson, A., Mbonu, J., Hussain, Z., Loh, W.-S. \& Fun, H.-K. (2015). Acta Cryst. E71, m139-m140.

Lu, X.-X., Luo, Y.-H., Lu, C., Chen, X. \& Zhang, H. (2015). J. Solid State Chem. 232, 123-130.

Macrae, C. F., Bruno, I. J., Chisholm, J. A., Edgington, P. R., McCabe, P., Pidcock, E., Rodriguez-Monge, L., Taylor, R., van de Streek, J. \& Wood, P. A. (2008). J. Appl. Cryst. 41, 466-470.

Mathews, I. I. \& Manohar, H. (1991). Acta Cryst. C47, 2213-2214.

Rujiwatra, A., Yimklan, S. \& Prior, T. J. (2012). Polyhedron, 31, 345351.

Segura, D. F., Netto, A. V. G., Frem, R. C. G., Mauro, A. E., da Silva, P. B., Fernandes, J. A., Paz, F. A. A., Dias, A. L. T., Silva, N. C., de Almeida, E. T., Marques, M. J., de Almeida, L., Alves, K. F., Pavan, F. R., de Souza, P. C., de Barros, H. B. \& Leite, C. Q. F. (2014). Polyhedron, 79, 197-206.

Sheldrick, G. M. (2008). Acta Cryst. A64, 112-122.
Table 2

Experimental details.

\begin{tabular}{ll}
\hline Crystal data & \\
Chemical formula & {$\left[\mathrm{Co}\left(\mathrm{NO}_{3}\right)\left(\mathrm{C}_{10} \mathrm{H}_{8} \mathrm{~N}_{2}\right)\left(\mathrm{H}_{2} \mathrm{O}\right)_{3}\right] \mathrm{Cl}$} \\
$M_{\mathrm{r}}$ & 366.62 \\
Crystal system, space group & Monoclinic, $P 2_{1} / c$ \\
Temperature $(\mathrm{K})$ & 295 \\
$a, b, c(\AA)$ & $11.5309(5), 8.9688(3), 14.7820(6)$ \\
$\beta\left({ }^{\circ}\right.$ & $111.915(3)$ \\
$V\left(\AA^{3}\right)$ & $1418.26(10)$ \\
$Z$ & 4 \\
Radiation type & $\mathrm{Ag} K \alpha, \lambda=0.56083 \AA$ \\
$\mu\left(\mathrm{mm}^{-1}\right)$ & 0.74 \\
Crystal size $(\mathrm{mm})$ & $0.60 \times 0.60 \times 0.60$ \\
& \\
Data collection & Stoe Stadivari \\
Diffractometer & Multi-scan $(X-A R E A$ and \\
Absorption correction & $X-R E D 32 ;$ Stoe \& Cie, 2015) \\
& $0.321,1.000$ \\
$T_{\text {min }}, T_{\text {max }}$ & $139350,9203,5851$ \\
No. of measured, independent and & \\
$\quad$ observed $[I>2 \sigma(I)]$ reflections & 0.068 \\
$R_{\text {int }}$ & 0.918 \\
(sin $\theta / \lambda)_{\text {max }}\left(\AA^{-1}\right)$ & \\
Refinement & \\
$R\left[F^{2}>2 \sigma\left(F^{2}\right)\right], w R\left(F^{2}\right), S$ & $0.045,0.140,1.10$ \\
No. of reflections & 9203 \\
No. of parameters & 214 \\
$\mathrm{H}$-atom treatment & $\mathrm{H}$ atoms treated by a mixture of \\
& independent and constrained \\
$\Delta \rho_{\text {max }}, \Delta \rho_{\text {min }}\left(\mathrm{e} \AA^{-3}\right)$ & refinement \\
\hline
\end{tabular}

Computer programs: X-AREA (Stoe \& Cie, 2015), SHELXT2014 (Sheldrick, 2015a), SHELXL2018 (Sheldrick, 2015b), XP in SHELXTL-Plus (Sheldrick, 2008), Mercury (Macrae et al., 2008) and publCIF (Westrip, 2010).

Sheldrick, G. M. (2015a). Acta Cryst. A71, 3-8.

Sheldrick, G. M. (2015b). Acta Cryst. C71, 3-8.

Stoe \& Cie (2015). $X$-AREA and $X$-RED32, Stoe \& Cie, Darmstadt, Germany.

Walmsley, F., Pinkerton, A. A. \& Walmsley, A. A. (1989). Polyhedron, 8, 689-693.

Westrip, S. P. (2010). J. Appl. Cryst. 43, 920-925.

Xiao, H.-P. (2006). Acta Cryst. E62, m95-m97.

Zhang, X.-F., Huang, D.-G., Wang, W.-G., Chen, C.-N. \& Liu, Q.-T. (2002). Acta Cryst. C58, m268-m269. 


\section{full crystallographic data}

IUCrData (2018). 3, x180866 [https://doi.org/10.1107/S2414314618008660]

fac-Triaqua $\left(2,2^{\prime}\right.$-bipyridine- $\left.\kappa^{2} N, N^{\prime}\right)($ nitrato- $\kappa O)$ cobalt(II) chloride

Mouhamadou Birame Diop, Libasse Diop and Sylvain Bernès

fac-Triaqua $\left(2,2^{\prime}\right.$-bipyridine- $\left.\kappa^{2} N, N^{\prime}\right)($ nitrato- $\kappa O)$ cobalt(II) chloride

Crystal data

$\left[\mathrm{Co}\left(\mathrm{NO}_{3}\right)\left(\mathrm{C}_{10} \mathrm{H}_{8} \mathrm{~N}_{2}\right)\left(\mathrm{H}_{2} \mathrm{O}\right)_{3}\right] \mathrm{Cl}$

$M_{r}=366.62$

Monoclinic, $P 2_{1} / c$

$a=11.5309(5) \AA$

$b=8.9688(3) \AA$

$c=14.7820(6) \AA$

$\beta=111.915(3)^{\circ}$

$V=1418.26(10) \AA^{3}$

$Z=4$

$F(000)=748$

$D_{\mathrm{x}}=1.717 \mathrm{Mg} \mathrm{m}^{-3}$

$\mathrm{Ag} K \alpha$ radiation, $\lambda=0.56083 \AA$

Cell parameters from 86540 reflections

$\theta=2.1-34.9^{\circ}$

$\mu=0.74 \mathrm{~mm}^{-1}$

$T=295 \mathrm{~K}$

Prism, orange

$0.60 \times 0.60 \times 0.60 \mathrm{~mm}$

\section{Data collection}

Stoe Stadivari diffractometer

Radiation source: Sealed X-ray tube, Axo Astix-

f Microfocus source

Graded multilayer mirror monochromator

Detector resolution: 5.81 pixels $\mathrm{mm}^{-1}$

$\omega$ scans

Absorption correction: multi-scan

(X-AREA and X-RED32; Stoe \& Cie, 2015)

Refinement

Refinement on $F^{2}$

Least-squares matrix: full

$R\left[F^{2}>2 \sigma\left(F^{2}\right)\right]=0.045$

$w R\left(F^{2}\right)=0.140$

$S=1.10$

9203 reflections

214 parameters

0 restraints

0 constraints

Primary atom site location: structure-invariant direct methods

$T_{\min }=0.321, T_{\max }=1.000$

139350 measured reflections

9203 independent reflections

5851 reflections with $I>2 \sigma(I)$

$R_{\text {int }}=0.068$

$\theta_{\text {max }}=31.0^{\circ}, \theta_{\text {min }}=2.1^{\circ}$

$h=-21 \rightarrow 21$

$k=-16 \rightarrow 16$

$l=-27 \rightarrow 22$

Secondary atom site location: difference Fourier map

Hydrogen site location: mixed

$\mathrm{H}$ atoms treated by a mixture of independent and constrained refinement

$w=1 /\left[\sigma^{2}\left(F_{\mathrm{o}}^{2}\right)+(0.0392 P)^{2}+1.4068 P\right]$ where $P=\left(F_{\mathrm{o}}^{2}+2 F_{\mathrm{c}}^{2}\right) / 3$

$(\Delta / \sigma)_{\max }<0.001$

$\Delta \rho_{\max }=1.28 \mathrm{e} \AA^{-3}$

$\Delta \rho_{\min }=-0.58$ e $\AA^{-3}$

Special details

Refinement. $\mathrm{H}$ atoms of the bipy ligand were placed in calculated positions and refined as riding to their carrier $\mathrm{C}$ atoms, with $U_{\text {iso }}=1.2 U_{\text {eq }}$ (carrier C). In contrast, water $\mathrm{H}$ atoms were refined with free coordinates and free $U_{\text {iso }}$ displacement parameters. $\mathrm{O}-\mathrm{H}$ bond lengths converged to acceptable values in the range 0.73 (3)-0.85 (4) $\AA$. 
Fractional atomic coordinates and isotropic or equivalent isotropic displacement parameters $\left(\AA^{2}\right)$

\begin{tabular}{|c|c|c|c|c|}
\hline & $x$ & $y$ & $z$ & $U_{\text {iso }} * / U_{\text {eq }}$ \\
\hline Co1 & $0.18156(2)$ & 0.28540 & $0.38779(2)$ & $0.02773(6)$ \\
\hline $\mathrm{Cl1}$ & $-0.05849(5)$ & $0.28742(6)$ & $0.06897(4)$ & $0.04189(11)$ \\
\hline N1 & $0.27083(15)$ & $0.43391(18)$ & $0.32300(11)$ & $0.0324(3)$ \\
\hline $\mathrm{C} 2$ & $0.2127(2)$ & $0.5247(2)$ & $0.24833(16)$ & $0.0422(4)$ \\
\hline $\mathrm{H} 2 \mathrm{~A}$ & 0.126113 & 0.532237 & 0.226040 & $0.051 *$ \\
\hline $\mathrm{C} 3$ & 0.2760 & $0.6083(3)$ & $0.20253(18)$ & $0.0491(5)$ \\
\hline $\mathrm{H} 3 \mathrm{~A}$ & 0.233048 & 0.669023 & 0.149601 & $0.059 *$ \\
\hline $\mathrm{C} 4$ & 0.4047 & 0.5986 & $0.23788(19)$ & $0.0508(5)$ \\
\hline $\mathrm{H} 4 \mathrm{~A}$ & 0.449930 & 0.654775 & 0.209499 & $0.061^{*}$ \\
\hline $\mathrm{C} 5$ & $0.4665(2)$ & $0.5053(2)$ & $0.31573(17)$ & 0.0409 (4) \\
\hline $\mathrm{H} 5 \mathrm{~A}$ & 0.553186 & 0.497382 & 0.339837 & $0.049 *$ \\
\hline C6 & $0.39652(17)$ & $0.4238(2)$ & $0.35692(13)$ & $0.0309(3)$ \\
\hline $\mathrm{C} 7$ & $0.45350(15)$ & $0.3213(2)$ & $0.44012(13)$ & 0.0299 \\
\hline $\mathrm{C} 8$ & $0.58164(17)$ & $0.2946(2)$ & $0.48387(16)$ & $0.0384(4)$ \\
\hline H8A & 0.636987 & 0.342089 & 0.460973 & $0.046^{*}$ \\
\hline C9 & $0.62560(19)$ & 0.1968 & $0.56154(17)$ & $0.0448(5)$ \\
\hline H9A & 0.710634 & 0.176436 & 0.590586 & $0.054 *$ \\
\hline $\mathrm{C} 10$ & $0.5423(2)$ & 0.1298 & $0.59550(17)$ & $0.0451(5)$ \\
\hline H10A & 0.570169 & 0.065224 & 0.648497 & $0.054 *$ \\
\hline C11 & $0.41622(18)$ & $0.1607(2)$ & $0.54913(15)$ & $0.0381(4)$ \\
\hline H11A & 0.359795 & 0.115400 & 0.571893 & $0.046^{*}$ \\
\hline N12 & $0.37249(13)$ & $0.25299(18)$ & $0.47297(11)$ & 0.0305 \\
\hline N13 & $0.21743(15)$ & $0.08151(19)$ & $0.22784(12)$ & 0.0339 \\
\hline O14 & $0.18036(16)$ & $0.09312(18)$ & $0.29758(11)$ & 0.0418 \\
\hline O15 & $0.2944(2)$ & $0.1667(2)$ & $0.21857(19)$ & $0.0689(6)$ \\
\hline O16 & $0.1752(2)$ & $-0.0228(2)$ & $0.16916(13)$ & $0.0560(5)$ \\
\hline $\mathrm{O} 1$ & $0.1557(2)$ & $0.4565(2)$ & $0.47721(14)$ & 0.0501 \\
\hline H11 & $0.120(4)$ & $0.539(5)$ & $0.455(3)$ & $0.077(11)^{*}$ \\
\hline H12 & $0.167(3)$ & $0.453(4)$ & $0.529(2)$ & $0.054(9)^{*}$ \\
\hline $\mathrm{O} 2$ & $0.12743(16)$ & $0.13141(19)$ & $0.46916(13)$ & 0.0415 \\
\hline $\mathrm{H} 21$ & $0.107(3)$ & $0.048(4)$ & $0.450(2)$ & $0.056(9)^{*}$ \\
\hline $\mathrm{H} 22$ & $0.085(3)$ & $0.153(4)$ & $0.494(3)$ & $0.070(11)^{*}$ \\
\hline $\mathrm{O} 3$ & $0.00497(15)$ & $0.3211(2)$ & $0.29102(13)$ & $0.0484(4)$ \\
\hline H31 & $-0.011(3)$ & $0.309(4)$ & $0.238(3)$ & $0.053(9)^{*}$ \\
\hline H32 & $-0.047(3)$ & $0.363(4)$ & $0.301(3)$ & $0.067(10)^{*}$ \\
\hline
\end{tabular}

Atomic displacement parameters $\left(\AA^{2}\right)$

\begin{tabular}{lllllll}
\hline & $U^{11}$ & $U^{22}$ & $U^{33}$ & $U^{12}$ & $U^{13}$ & $U^{23}$ \\
\hline Co1 & $0.02527(9)$ & $0.03019(11)$ & $0.02956(10)$ & $0.00029(8)$ & $0.01235(7)$ & $0.00041(8)$ \\
C11 & $0.0487(3)$ & $0.0382(2)$ & $0.0462(2)$ & $-0.00178(19)$ & $0.0263(2)$ & $-0.00160(19)$ \\
N1 & $0.0344(7)$ & $0.0314(6)$ & $0.0322(6)$ & $-0.0008(5)$ & $0.0135(5)$ & $0.0019(5)$ \\
C2 & $0.0462(10)$ & $0.0392(9)$ & $0.0380(9)$ & $-0.0015(8)$ & $0.0120(8)$ & $0.0065(8)$ \\
C3 & $0.0668(15)$ & $0.0416(11)$ & $0.0417(10)$ & $-0.0021(10)$ & $0.0233(10)$ & $0.0088(8)$ \\
C4 & $0.0696(15)$ & $0.0440(11)$ & $0.0518(12)$ & $-0.0087(11)$ & $0.0376(12)$ & $0.0051(9)$
\end{tabular}




$\begin{array}{lllllll}\text { C5 } & 0.0426(9) & 0.0408(9) & 0.0484(10) & -0.0071(8) & 0.0276(9) & -0.0009(8) \\ \text { C6 } & 0.0350(7) & 0.0298(7) & 0.0327(7) & -0.0044(6) & 0.0181(6) & -0.0032(6) \\ \text { C7 } & 0.0265(6) & 0.0320(7) & 0.0333(7) & -0.0028(5) & 0.0136(6) & -0.0051(6) \\ \text { C8 } & 0.0246(6) & 0.0460(10) & 0.0456(10) & -0.0023(7) & 0.0141(6) & -0.0072(8) \\ \text { C9 } & 0.0270(7) & 0.0536(12) & 0.0483(11) & 0.0070(8) & 0.0076(7) & -0.0035(9) \\ \text { C10 } & 0.0354(9) & 0.0503(12) & 0.0426(10) & 0.0078(8) & 0.0065(8) & 0.0089(9) \\ \text { C11 } & 0.0331(8) & 0.0432(10) & 0.0369(8) & 0.0022(7) & 0.0120(7) & 0.0070(7) \\ \text { N12 } & 0.0257(5) & 0.0348(6) & 0.0310(6) & -0.0006(5) & 0.0107(5) & 0.0015(5) \\ \text { N13 } & 0.0329(7) & 0.0353(7) & 0.0362(7) & 0.0012(5) & 0.0159(6) & -0.0047(6) \\ \text { O14 } & 0.0543(9) & 0.0399(7) & 0.0392(7) & -0.0043(6) & 0.0267(7) & -0.0068(6) \\ \text { O15 } & 0.0752(13) & 0.0606(11) & 0.0999(17) & -0.0284(10) & 0.0662(13) & -0.0288(11) \\ \text { O16 } & 0.0763(12) & 0.0543(10) & 0.0487(9) & -0.0240(9) & 0.0363(9) & -0.0226(8) \\ \text { O1 } & 0.0793(13) & 0.0393(8) & 0.0397(8) & 0.0133(8) & 0.0316(8) & 0.0000(6) \\ \text { O2 } & 0.0482(8) & 0.0363(7) & 0.0526(9) & -0.0060(6) & 0.0336(7) & -0.0005(6) \\ \text { O3 } & 0.0321(7) & 0.0713(12) & 0.0386(8) & 0.0144(7) & 0.0096(6) & -0.0044(8) \\ & & & & & \end{array}$

Geometric parameters $\left(A,{ }^{\circ}\right)$

\begin{tabular}{|c|c|c|c|}
\hline $\mathrm{Co} 1-\mathrm{O} 3$ & $2.0300(16)$ & $\mathrm{C} 7-\mathrm{C} 8$ & $1.395(2)$ \\
\hline $\mathrm{Co} 1-\mathrm{O} 2$ & $2.0746(15)$ & $\mathrm{C} 8-\mathrm{C} 9$ & $1.382(3)$ \\
\hline $\mathrm{Co} 1-\mathrm{N} 12$ & $2.1090(15)$ & $\mathrm{C} 8-\mathrm{H} 8 \mathrm{~A}$ & 0.9300 \\
\hline $\mathrm{Co} 1-\mathrm{O} 1$ & $2.1177(16)$ & $\mathrm{C} 9-\mathrm{C} 10$ & $1.377(3)$ \\
\hline $\mathrm{Co} 1-\mathrm{N} 1$ & $2.1183(15)$ & C9-H9A & 0.9300 \\
\hline $\mathrm{Co} 1-\mathrm{O} 14$ & $2.1769(15)$ & $\mathrm{C} 10-\mathrm{C} 11$ & $1.384(3)$ \\
\hline $\mathrm{N} 1-\mathrm{C} 2$ & $1.333(3)$ & $\mathrm{C} 10-\mathrm{H} 10 \mathrm{~A}$ & 0.9300 \\
\hline $\mathrm{N} 1-\mathrm{C} 6$ & $1.348(2)$ & $\mathrm{C} 11-\mathrm{N} 12$ & $1.335(2)$ \\
\hline $\mathrm{C} 2-\mathrm{C} 3$ & $1.386(3)$ & C11-H11A & 0.9300 \\
\hline $\mathrm{C} 2-\mathrm{H} 2 \mathrm{~A}$ & 0.9300 & $\mathrm{~N} 13-\mathrm{O} 15$ & $1.217(2)$ \\
\hline $\mathrm{C} 3-\mathrm{C} 4$ & $1.380(4)$ & $\mathrm{N} 13-\mathrm{O} 16$ & $1.245(2)$ \\
\hline $\mathrm{C} 3-\mathrm{H} 3 \mathrm{~A}$ & 0.9300 & $\mathrm{~N} 13-\mathrm{O} 14$ & $1.259(2)$ \\
\hline $\mathrm{C} 4-\mathrm{C} 5$ & $1.386(4)$ & $\mathrm{O} 1-\mathrm{H} 11$ & $0.85(4)$ \\
\hline $\mathrm{C} 4-\mathrm{H} 4 \mathrm{~A}$ & 0.9300 & $\mathrm{O} 1-\mathrm{H} 12$ & $0.73(3)$ \\
\hline $\mathrm{C} 5-\mathrm{C} 6$ & $1.387(3)$ & $\mathrm{O} 2-\mathrm{H} 21$ & $0.81(4)$ \\
\hline $\mathrm{C} 5-\mathrm{H} 5 \mathrm{~A}$ & 0.9300 & $\mathrm{O} 2-\mathrm{H} 22$ & $0.74(4)$ \\
\hline $\mathrm{C} 6-\mathrm{C} 7$ & $1.479(3)$ & $\mathrm{O} 3-\mathrm{H} 31$ & $0.74(4)$ \\
\hline $\mathrm{C} 7-\mathrm{N} 12$ & $1.350(2)$ & $\mathrm{O} 3-\mathrm{H} 32$ & $0.77(4)$ \\
\hline $\mathrm{O} 3-\mathrm{Co} 1-\mathrm{O} 2$ & $94.24(8)$ & $\mathrm{C} 5-\mathrm{C} 6-\mathrm{C} 7$ & $122.82(18)$ \\
\hline $\mathrm{O} 3-\mathrm{Co} 1-\mathrm{N} 12$ & $172.53(7)$ & $\mathrm{N} 12-\mathrm{C} 7-\mathrm{C} 8$ & $120.71(18)$ \\
\hline $\mathrm{O} 2-\mathrm{Co} 1-\mathrm{N} 12$ & $92.01(7)$ & $\mathrm{N} 12-\mathrm{C} 7-\mathrm{C} 6$ & $115.41(15)$ \\
\hline $\mathrm{O} 3-\mathrm{Co} 1-\mathrm{O} 1$ & $89.28(8)$ & $\mathrm{C} 8-\mathrm{C} 7-\mathrm{C} 6$ & $123.88(16)$ \\
\hline $\mathrm{O} 2-\mathrm{Co} 1-\mathrm{O} 1$ & $88.72(7)$ & $\mathrm{C} 9-\mathrm{C} 8-\mathrm{C} 7$ & $119.40(19)$ \\
\hline $\mathrm{N} 12-\mathrm{Co} 1-\mathrm{O} 1$ & $94.94(8)$ & $\mathrm{C} 9-\mathrm{C} 8-\mathrm{H} 8 \mathrm{~A}$ & 120.3 \\
\hline $\mathrm{O} 3-\mathrm{Co} 1-\mathrm{N} 1$ & $96.37(7)$ & $\mathrm{C} 7-\mathrm{C} 8-\mathrm{H} 8 \mathrm{~A}$ & 120.3 \\
\hline $\mathrm{O} 2-\mathrm{Co} 1-\mathrm{N} 1$ & $169.37(7)$ & $\mathrm{C} 10-\mathrm{C} 9-\mathrm{C} 8$ & $119.36(18)$ \\
\hline $\mathrm{N} 12-\mathrm{Co} 1-\mathrm{N} 1$ & $77.37(6)$ & $\mathrm{C} 10-\mathrm{C} 9-\mathrm{H} 9 \mathrm{~A}$ & 120.3 \\
\hline $\mathrm{O} 1-\mathrm{Co} 1-\mathrm{N} 1$ & $91.83(7)$ & $\mathrm{C} 8-\mathrm{C} 9-\mathrm{H} 9 \mathrm{~A}$ & 120.3 \\
\hline $\mathrm{O} 3-\mathrm{Co} 1-\mathrm{O} 14$ & $85.30(7)$ & $\mathrm{C} 9-\mathrm{C} 10-\mathrm{C} 11$ & $118.6(2)$ \\
\hline
\end{tabular}




\begin{tabular}{|c|c|c|c|}
\hline $\mathrm{O} 2-\mathrm{Co} 1-\mathrm{O} 14$ & $83.87(6)$ & $\mathrm{C} 9-\mathrm{C} 10-\mathrm{H} 10 \mathrm{~A}$ & 120.7 \\
\hline $\mathrm{N} 12-\mathrm{Co} 1-\mathrm{O} 14$ & $91.32(6)$ & $\mathrm{C} 11-\mathrm{C} 10-\mathrm{H} 10 \mathrm{~A}$ & 120.7 \\
\hline $\mathrm{O} 1-\mathrm{Co1}-\mathrm{O} 14$ & $170.47(7)$ & $\mathrm{N} 12-\mathrm{C} 11-\mathrm{C} 10$ & $122.52(19)$ \\
\hline $\mathrm{N} 1-\mathrm{Co} 1-\mathrm{O} 14$ & $96.56(6)$ & $\mathrm{N} 12-\mathrm{C} 11-\mathrm{H} 11 \mathrm{~A}$ & 118.7 \\
\hline $\mathrm{C} 2-\mathrm{N} 1-\mathrm{C} 6$ & $119.15(17)$ & $\mathrm{C} 10-\mathrm{C} 11-\mathrm{H} 11 \mathrm{~A}$ & 118.7 \\
\hline $\mathrm{C} 2-\mathrm{N} 1-\mathrm{Co} 1$ & $125.40(14)$ & $\mathrm{C} 11-\mathrm{N} 12-\mathrm{C} 7$ & $119.36(16)$ \\
\hline $\mathrm{C} 6-\mathrm{N} 1-\mathrm{Co} 1$ & $115.22(12)$ & $\mathrm{C} 11-\mathrm{N} 12-\mathrm{Co} 1$ & $124.77(13)$ \\
\hline $\mathrm{N} 1-\mathrm{C} 2-\mathrm{C} 3$ & $122.8(2)$ & $\mathrm{C} 7-\mathrm{N} 12-\mathrm{Co} 1$ & $115.53(12)$ \\
\hline $\mathrm{N} 1-\mathrm{C} 2-\mathrm{H} 2 \mathrm{~A}$ & 118.6 & $\mathrm{O} 15-\mathrm{N} 13-\mathrm{O} 16$ & $120.34(18)$ \\
\hline $\mathrm{C} 3-\mathrm{C} 2-\mathrm{H} 2 \mathrm{~A}$ & 118.6 & $\mathrm{O} 15-\mathrm{N} 13-\mathrm{O} 14$ & $121.67(18)$ \\
\hline $\mathrm{C} 4-\mathrm{C} 3-\mathrm{C} 2$ & $118.0(2)$ & $\mathrm{O} 16-\mathrm{N} 13-\mathrm{O} 14$ & $117.96(17)$ \\
\hline $\mathrm{C} 4-\mathrm{C} 3-\mathrm{H} 3 \mathrm{~A}$ & 121.0 & $\mathrm{~N} 13-\mathrm{O} 14-\mathrm{Co} 1$ & $129.84(13)$ \\
\hline $\mathrm{C} 2-\mathrm{C} 3-\mathrm{H} 3 \mathrm{~A}$ & 121.0 & $\mathrm{Co} 1-\mathrm{O} 1-\mathrm{H} 11$ & $124(3)$ \\
\hline $\mathrm{C} 3-\mathrm{C} 4-\mathrm{C} 5$ & $120.0(2)$ & $\mathrm{Co} 1-\mathrm{O} 1-\mathrm{H} 12$ & $128(3)$ \\
\hline $\mathrm{C} 3-\mathrm{C} 4-\mathrm{H} 4 \mathrm{~A}$ & 120.0 & $\mathrm{H} 11-\mathrm{O} 1-\mathrm{H} 12$ & $108(4)$ \\
\hline $\mathrm{C} 5-\mathrm{C} 4-\mathrm{H} 4 \mathrm{~A}$ & 120.0 & $\mathrm{Co} 1-\mathrm{O} 2-\mathrm{H} 21$ & $122(2)$ \\
\hline $\mathrm{C} 4-\mathrm{C} 5-\mathrm{C} 6$ & $118.7(2)$ & $\mathrm{Co} 1-\mathrm{O} 2-\mathrm{H} 22$ & $121(3)$ \\
\hline $\mathrm{C} 4-\mathrm{C} 5-\mathrm{H} 5 \mathrm{~A}$ & 120.7 & $\mathrm{H} 21-\mathrm{O} 2-\mathrm{H} 22$ & $105(4)$ \\
\hline $\mathrm{C} 6-\mathrm{C} 5-\mathrm{H} 5 \mathrm{~A}$ & 120.7 & $\mathrm{Co} 1-\mathrm{O} 3-\mathrm{H} 31$ & $121(3)$ \\
\hline $\mathrm{N} 1-\mathrm{C} 6-\mathrm{C} 5$ & $121.50(18)$ & $\mathrm{Co} 1-\mathrm{O} 3-\mathrm{H} 32$ & $126(3)$ \\
\hline $\mathrm{N} 1-\mathrm{C} 6-\mathrm{C} 7$ & $115.68(14)$ & $\mathrm{H} 31-\mathrm{O} 3-\mathrm{H} 32$ & $112(4)$ \\
\hline $\mathrm{C} 6-\mathrm{N} 1-\mathrm{C} 2-\mathrm{C} 3$ & $1.0(3)$ & $\mathrm{C} 5-\mathrm{C} 6-\mathrm{C} 7-\mathrm{C} 8$ & $-1.2(3)$ \\
\hline $\mathrm{Co} 1-\mathrm{N} 1-\mathrm{C} 2-\mathrm{C} 3$ & $-173.21(18)$ & $\mathrm{N} 12-\mathrm{C} 7-\mathrm{C} 8-\mathrm{C} 9$ & $0.1(3)$ \\
\hline $\mathrm{N} 1-\mathrm{C} 2-\mathrm{C} 3-\mathrm{C} 4$ & $-1.5(4)$ & $\mathrm{C} 6-\mathrm{C} 7-\mathrm{C} 8-\mathrm{C} 9$ & $179.84(19)$ \\
\hline $\mathrm{C} 2-\mathrm{C} 3-\mathrm{C} 4-\mathrm{C} 5$ & $1.2(4)$ & $\mathrm{C} 7-\mathrm{C} 8-\mathrm{C} 9-\mathrm{C} 10$ & $-1.3(3)$ \\
\hline $\mathrm{C} 3-\mathrm{C} 4-\mathrm{C} 5-\mathrm{C} 6$ & $-0.5(4)$ & $\mathrm{C} 8-\mathrm{C} 9-\mathrm{C} 10-\mathrm{C} 11$ & $1.3(4)$ \\
\hline $\mathrm{C} 2-\mathrm{N} 1-\mathrm{C} 6-\mathrm{C} 5$ & $-0.2(3)$ & $\mathrm{C} 9-\mathrm{C} 10-\mathrm{C} 11-\mathrm{N} 12$ & $-0.1(4)$ \\
\hline $\mathrm{Co} 1-\mathrm{N} 1-\mathrm{C} 6-\mathrm{C} 5$ & $174.59(15)$ & $\mathrm{C} 10-\mathrm{C} 11-\mathrm{N} 12-\mathrm{C} 7$ & $-1.0(3)$ \\
\hline $\mathrm{C} 2-\mathrm{N} 1-\mathrm{C} 6-\mathrm{C} 7$ & $179.69(17)$ & $\mathrm{C} 10-\mathrm{C} 11-\mathrm{N} 12-\mathrm{Co} 1$ & $172.08(18)$ \\
\hline $\mathrm{Co} 1-\mathrm{N} 1-\mathrm{C} 6-\mathrm{C} 7$ & $-5.5(2)$ & $\mathrm{C} 8-\mathrm{C} 7-\mathrm{N} 12-\mathrm{C} 11$ & $1.0(3)$ \\
\hline $\mathrm{C} 4-\mathrm{C} 5-\mathrm{C} 6-\mathrm{N} 1$ & $-0.1(3)$ & $\mathrm{C} 6-\mathrm{C} 7-\mathrm{N} 12-\mathrm{C} 11$ & $-178.73(17)$ \\
\hline $\mathrm{C} 4-\mathrm{C} 5-\mathrm{C} 6-\mathrm{C} 7$ & $-179.96(19)$ & $\mathrm{C} 8-\mathrm{C} 7-\mathrm{N} 12-\mathrm{Co} 1$ & $-172.70(14)$ \\
\hline $\mathrm{N} 1-\mathrm{C} 6-\mathrm{C} 7-\mathrm{N} 12$ & $-1.3(2)$ & $\mathrm{C} 6-\mathrm{C} 7-\mathrm{N} 12-\mathrm{Co} 1$ & $7.5(2)$ \\
\hline $\mathrm{C} 5-\mathrm{C} 6-\mathrm{C} 7-\mathrm{N} 12$ & $178.58(18)$ & $\mathrm{O} 15-\mathrm{N} 13-\mathrm{O} 14-\mathrm{Co} 1$ & $22.7(3)$ \\
\hline $\mathrm{N} 1-\mathrm{C} 6-\mathrm{C} 7-\mathrm{C} 8$ & $178.93(17)$ & $\mathrm{O} 16-\mathrm{N} 13-\mathrm{O} 14-\mathrm{Co} 1$ & $-159.05(17)$ \\
\hline
\end{tabular}

Hydrogen-bond geometry $\left(\AA,{ }^{\circ}\right)$

\begin{tabular}{lllll}
\hline$D-\mathrm{H} \cdots A$ & $D-\mathrm{H}$ & $\mathrm{H} \cdots A$ & $D \cdots A$ & $D-\mathrm{H} \cdots A$ \\
\hline $\mathrm{C} 11-\mathrm{H} 11 A \cdots \mathrm{O} 2$ & 0.93 & 2.54 & $3.101(3)$ & 119 \\
$\mathrm{O} 1-\mathrm{H} 11 \cdots \mathrm{C} 11^{\mathrm{i}}$ & $0.85(4)$ & $2.33(4)$ & $3.1560(19)$ & $166(4)$ \\
$\mathrm{O} 1-\mathrm{H} 12 \cdots \mathrm{O} 16^{\mathrm{ii}}$ & $0.73(3)$ & $2.13(3)$ & $2.825(2)$ & $160(4)$ \\
$\mathrm{O} 2-\mathrm{H} 21 \cdots \mathrm{C} 11^{i i}$ & $0.81(4)$ & $2.39(4)$ & $3.1833(18)$ & $167(3)$ \\
$\mathrm{O} 2-\mathrm{H} 22 \cdots \mathrm{C} 11^{\mathrm{ii}}$ & $0.74(4)$ & $2.37(4)$ & $3.1088(16)$ & $177(4)$
\end{tabular}




\section{data reports}

$\mathrm{O} 3-\mathrm{H} 31 \cdots \mathrm{Cl} 1$

$0.74(4)$

$0.77(4)$

$2.36(4)$

$3.100(2)$

$176(3)$

$\mathrm{O} 3-\mathrm{H} 32 \cdots \mathrm{O} 16$

$1.98(4)$

$2.745(2)$

$178(4)$

Symmetry codes: (i) $-x, y+1 / 2,-z+1 / 2$; (ii) $x,-y+1 / 2, z+1 / 2$; (iii) $-x, y-1 / 2,-z+1 / 2$. 